\title{
Sicherungsverwahrung ist ein Thema für die Psychiatrie - Pro
}

\author{
Preventive Detention is a Psychiatric Issue - Pro
}

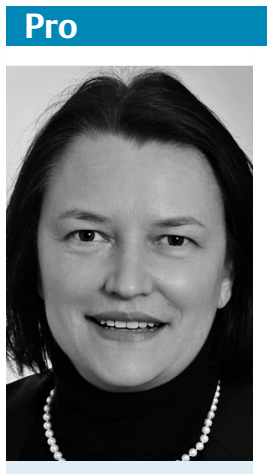

Manuela Dudeck

Die Sicherungsver-

wahrung ist spätestens seit der Rechtssache $M$. gegen Deutschland (Beschwerde-Nr. 19359/ 04) in den Fokus der Öffentlichkeit gerückt, nachdem ein Ausschuss von fünf Richtern in seiner Sitzung am 10. Mai 2010 den Antrag der deutschen Bundesregierung auf Verweisung an die Große Kammer vom 16. März 2010 abgelehnt hat. Damit war und ist das Kammerurteil des Gerichtshofs in dieser Sache vom 17. Dezember 2009 rechtskräftig (Artikel 44 der Europäischen Menschenrechtskonvention). Der Fall betraf die rückwirkende Verlängerung der Sicherungsverwahrung eines in Expertengutachten als gefährlich eingestuften Straftäters über die zum Zeitpunkt seiner Verurteilung maximal zulässige Höchstdauer von zehn Jahren hinaus. In seinem Kammerurteil vom 17. Dezember 2009 kam der Europäische Gerichtshof einstimmig zu dem Schluss, dass darin eine Verletzung von Artikel $5 \S 1$ (Recht auf Freiheit) und Artikel $7 \S 1$ (keine Strafe ohne Gesetz) der Konvention vorlag. Gleichzeitig unterstrich der Europäische Gerichtshof, dass die Sicherungsverwahrung des Beschwerdeführers vor Ablauf der Zehnjahresfrist zulässig war. Daraufhin erklärte das Bundesverfassungsgericht mit seinem Urteil von 4. Mai 2011 fast alle Vorschriften zur Sicherungsverwahrung mit mehreren Grundgesetzartikeln als einander ausschließend (2 BvR 2365/09, 2 BvR 740/10, 2 BvR 2333/08, 2 BvR 1152/10, 2 BvR 571/10).

Das darauffolgende Gesetz zur Therapierung und Unterbringung psychisch gestörter Gewalttäter (Therapieunterbringungsgesetz - ThUG) vom 1. November 2011 hatte vor allem kritische Kommentare zur Folge. Dennoch war in diesem Zu- sammenhang die Definition des zentralen Tatbestandsmerkmals „unsound mind“ gefragt, welches nach der Rechtsprechung des Europäischen Gerichtshofs für Menschenrechte eine zuverlässig nachgewiesene psychische Störung („true mental disorder“) voraussetzt, die eine zwangsweise Unterbringung erfordert („warranting compulsory confinement“), und die fortdauert (,the validity of continued confinement must depend upon the persistence of such a disorder"). Damit waren Psychiaterinnen und Psychiater gefordert, die psychische Befindlichkeit eines Sicherungsverwahrten zu konkretisieren und interdisziplinär zu thematisieren.

Gairing und Kollegen definierten den Sicherungsverwahrten als Menschen im fortgeschrittenen Alter mit antisozialen Persönlichkeitszügen, welcher neben Substanzmissbrauch und Aggressivität über viele Vorstrafen und Haftjahre verfügt und somit mehr Überschneidungen mit einem Gefängnisinsassen als mit einem Maßregelpatienten aufweist [1]. Die Forschungsliteratur der vergangenen 20 Jahre zur psychischen Belastung von Gefangenen legt den Schluss nahe, dass diese hoch und nicht ausreichend behandelt ist. Am häufigsten finden sich neben affektiven und Angsterkrankungen die substanzbezogenen Störungen mit bis zu $68 \%$. In der Metaanalyse von Fazel und Danesh wurden auch psychotische Störungen in einem Umfang von 4\% gefunden [2]. Die mit den o.g. Erkrankungen komorbiden Persönlichkeitsstörungen haben dabei eine Auftretenshäufigkeit von $50-80 \%$. Führend sind zu 75\% die antisoziale Persönlichkeitsstörung und nachfolgend die emotional-instabile Persönlichkeitsstörung vom Borderline- bzw. impulsiven Typus [3]. Bereits bei jugendlichen und heranwachsenden Straftätern ergibt sich eine daraus resultierende psychische Belastung, welche der einer klinischen Gruppe von Patienten mit Persönlichkeitsstörungen entspricht [4]. Vergleicht man Gefängnisinsassen hinsichtlich der Haftlänge, so bildet sich bei Langzeitge- fangenen ein psychiatrischer Behandlungsbedarf bei $65 \%$ und bei Kurzzeitgefangenen bei $40 \%$ ab, womit das Vorliegen einer auffälligen psychischen Gesamtbelastung bei den Langzeitgefangenen um mehr als 50\% erhöht ist [5]. Diese Ergebnisse bedeuten eine klare Annäherung an die Kennwerte klinischer Populationen und sind Thema der Psychiatrie [6].

Die Ursachen für die hohe Prävalenz psychischer Störungsbilder bei Gefängnisinsassen des Strafvollzugs sind vielfältig: Psychische Erkrankungen und traumatische Erfahrungen können bereits vor Antritt der Haft vorhanden gewesen sein. So waren mindestens 30\% der Straftäter vor Inhaftierung in psychiatrischer Behandlung, und jeder sechste zeigte in der Anamnese selbstverletzendes und/oder suizidales Verhalten [7]. Andererseits kann der Freiheitsentzug unter möglicherweise ungünstigen Haftbedingungen und ohne hinreichende psychiatrische Versorgung dazu beitragen, dass sich das psychische Befinden auch während der Haft verschlechtert. Namentlich chronisch psychisch Kranke fallen zunächst im Alltag eines Gefängnisses weniger auf und werden seltener einem internen oder externen Psychiater vorgestellt. Aufgrund dessen verschlimmern sich psychische Störungsbilder, welche nicht erkannt und behandelt werden, erhöhen das individuelle Leid der Inhaftierten, führen zu interaktionellen Schwierigkeiten mit dem Gefängnispersonal und anderen Gefangenen und stellen möglicherweise einen Risikofaktor für weitere psychische Erkrankungen, selbstverletzendes oder suizidales Verhalten dar. Es kann davon ausgegangen werden, dass psychische Erkrankungen eine konstruktive Auseinandersetzung mit dem eigenen Delikt erschweren und somit nicht zu einer Senkung des Rückfallrisikos beitragen. Insbesondere das Auftreten von Substanzabhängigkeiten und Persönlichkeitsstörungen ist signifikant mit einem erhöhten Rückfallrisiko assoziiert, und so verwundert es nicht, dass behandelte Straftäter 
signifikant seltener rückfällig werden und positivere Verläufe als unbehandelte Straftäter zeigen $[8,9]$.

Nicht zuletzt mindert das Vorhandensein psychischer Erkrankungen die Wahrscheinlichkeit der sozialen Reintegration. Damit ist das Thema nicht nur von psychiatrischer, sondern auch von volkswirtschaftlicher, gesellschaftlicher und ethischer Relevanz. Nicht umsonst geben die Empfehlungen des Europarates an seine Mitgliedstaaten vor, für psychisch erkrankte Gefängnisinsassen dieselben Behandlungsstandards wie für die Allgemeinbevölkerung vorzuhalten (European Prison Rules 40.2; 40.4; 47.1) [10].

Zusammenfassend handelt es sich bei Sicherungsverwahrten um eine Klientel, die angesichts hochkomplexer Störungsbilder in Verbindung mit aggressivem Verhalten schwer therapierbar sind. Therapiepessimismus scheint dennoch nicht angebracht, da mittlerweile gut evaluierte Therapieprogramme und Rehabilitationsmodelle für Straftäter existieren.

\section{Literatur}

1 Gairing SK, de Tribolet-Hardy F, Vohs $K$ et al. Sicherungsverwahrte ( $\$ 66$ StGB). Merkmale der Täter und ihre Bedeutung für die Erfolgsaussichten eines therapeutischen Vollzugs. Nervenarzt 2013; 84: 65-71

2 Fazel S, Danesh J. Serious mental disorders in 23000 prisoners: a systematic review of 62 surveys. Lancet 2002; 16: 545-550

3 Dudeck M, Kopp D, Drenkhahn $K$ et al. Die Prävalenz psychischer Erkrankungen bei Gefängnisinsassen mit Kurzzeitstrafe. Psych Prax 2009; 36: 1-6

4 Köhler D, Hinrichs G, Baving $L$. Therapiemotivation, Psychische Belastung und Persönlichkeit bei Inhaftierten des Jugendvollzuges. Z Klin Psychol Psychiatr Psychother 2008; 37: 24-31

5 Kopp D, Drenkhahn K, Dünkel F et al. Psychische Symptombelastung bei Kurz- und Langzeitstrafgefangenen in Deutschland. Nervenarzt 2011; 82: 880-885

6 Franke GH. SCL-90-R. Die Symptom-Checkliste von Derogates - Deutsche Version Manual. Göttingen: Beltz Test GmbH; 1995

7 Dudeck M, Drenkhahn K, Spitzer C et al. Traumatization and mental distress in long-term prisoners in Europe. Punishment \& Society 2011; 13: 403-423

8 Grann M, Danesh J, Fazel S. The association between psychiatric diagnosis and violent reoffending in adult offenders in the community. BMC Psychiatry10.1186/1471244X-8-92
9 McGire J. Integrating Findigs from Research Reviews. In: McGire J, ed. Offender Rehabilitation and Treatment: Effective Programmes and Policies to Reduce Reoffending. West Sussex, UK: John Wiley \& Sons; 2002: 3-38

10 Empfehlung Rec(2006)2 des Ministerkomitees an die Mitgliedstaaten über die Europäischen Strafvollzugsgrundsätze. Im Internet: https://wcd.coe.int/ViewDoc.jsp?id= 955747

Sie haben eine eigene Meinung zu diesem

Thema? Dann schreiben Sie uns an:

psychiat-praxis@thieme.de!

\section{Korrespondenzadresse}

\section{Univ.-Prof. Dr. med. Manuela Dudeck}

Ärztliche Direktorin der Klinik für Forensische Psychiatrie und Psychotherapie der Universität Ulm am BKH Günzburg

Ludwig-Heilmeyer-Straße 2/Haus 58 89312 Günzburg

Manuela.Dudeck@bkh-guenzburg.de

\section{Bibliografie}

DOI http://dx.doi.org/

10.1055/s-0034-1387337

Psychiat Prax 2014; 41: 352-353

(c) Georg Thieme Verlag KG

Stuttgart · New York

ISSN 0303-4259 